\title{
Adding many Baumgartner clubs
}

\author{
David Asperó ${ }^{1}$
}

Received: 5 February 2014 / Accepted: 23 August 2014 / Published online: 9 May 2017

(C) The Author(s) 2017. This article is an open access publication

Abstract I define a homogeneous $\aleph_{2}-$ c.c. proper product forcing for adding many clubs of $\omega_{1}$ with finite conditions. I use this forcing to build models of $\mathfrak{b}\left(\omega_{1}\right)=\aleph_{2}$, together with $\mathfrak{d}\left(\omega_{1}\right)$ and $2^{\aleph_{0}}$ large and with very strong failures of club guessing at $\omega_{1}$.

Keywords Baumgartner clubs - Strong failures of club guessing - Cardinal characteristics for $\omega_{1} \cdot$ Generalizations of Martin's Axiom

Mathematics Subject Classification 03E05 - 03E35 - 03E50 - 03E17

\section{Introduction}

Cohen's forcing $2^{<\omega}$ for adding a real, usually called Cohen forcing, is perhaps the simplest non-trivial forcing notion one can think of (and it was also the first to be discovered). There is a very simple and nicely behaved forcing for adding an arbitrary number $\theta$ of Cohen reals. This forcing is of course $\operatorname{Add}(\omega, \theta)$, where $\operatorname{Add}(\omega, X)$, for a set of ordinals $X$, is the partial order of finite functions $p \subseteq X \times \omega \times 2$, ordered by reverse inclusion. For every $\operatorname{Add}(\omega, X)$-generic $G$ and every $\alpha \in X$, $r_{\alpha}^{G}:=\bigcup_{p \in G}\{(n, \epsilon) \mid(\alpha, n, \epsilon) \in p\}$ is a Cohen real over $\mathbf{V}$ and $r_{\alpha}^{G} \neq r_{\alpha^{\prime}}^{G}$ for $\alpha \neq \alpha^{\prime}$ in $X$. Also, $\operatorname{Add}(\omega, X)$ is nicely behaved: It has the countable chain condition (c.c.c.), it is homogeneous - in the sense that given any $p, p^{\prime} \in \operatorname{Add}(\omega, X)$ there are $q \leq p$ and $q^{\prime} \leq p^{\prime}$ such that $\operatorname{Add}(\omega, X) \uparrow q \cong \operatorname{Add}(\omega, X) \uparrow q^{\prime}-$, and it can be naturally represented as the product of $\operatorname{Add}\left(\omega, X_{0}\right)$ and $\operatorname{Add}\left(\omega, X_{1}\right)$ for any partition $\left(X_{0}, X_{1}\right)$ of $X$. In particular, for every $G$ as above and all $\alpha \neq \alpha^{\prime}$ in $X, r_{\alpha^{\prime}}^{G}$ is Cohen generic

$\bowtie$ David Asperó

d.aspero@uea.ac.uk

1 School of Mathematics, University of East Anglia, Norwich NR4 7TJ, UK 
over $\mathbf{V}\left[r_{\alpha}^{G}\right]$. Cohen forcing and $\operatorname{Add}(\omega, \theta)$ have of course been extensively studied for more than 50 years now. For example, $\operatorname{Add}(\omega, \theta)$ is the forcing that Cohen used to prove the consistency of $\neg \mathrm{CH}$ (by forcing over $L$ ).

There is a particularly simple forcing notion for adding a club subset of $\omega_{1}$. This forcing was first defined and studied by Baumgartner [4] and will be denoted in this note by $\mathbb{B}$. $\mathbb{B}$ is the set, ordered by reverse inclusion, of all finite functions $f \subseteq \omega_{1} \times \omega_{1}$ that can be extended to a strictly increasing and continuous function $F: \omega_{1} \longrightarrow \omega_{1}$. $\mathbb{B}$ has size $\aleph_{1}$, and the union of any generic filter for $\mathbb{B}$ is the enumerating function of a new club of $\omega_{1}$, which I will call a Baumgartner club (over $\mathbf{V}$ ). $\mathbb{B}$ is often described as the forcing for adding a club of $\omega_{1}$ with finite conditions. It can be presented in other appealing ways too (see for example [1] or [16]). $\mathbb{B}$ is proper and so, since it has size $\aleph_{1}$, preserves all cardinals; in fact, given any countable $N \preccurlyeq H\left(\omega_{2}\right)$ and any $p \in \mathbb{B} \cap N, p^{*}:=p \cup\left\{\left\langle\delta_{N}, \delta_{N}\right\rangle\right\}$ is an $(N, \mathbb{B})$-generic condition extending $p$ (see [4]) where, here and throughout the note, $\delta_{X}$ denotes $X \cap \omega_{1}$ whenever $X$ is a set and $X \cap \omega_{1}$ is an ordinal. The proof of properness for $\mathbb{B}$ coming from this choice of $p^{*}$ can in fact be seen as perhaps the simplest possible proof of properness of a partial order using submodels as side conditions: $N$ is 'added' to $p$ by declaring $\delta_{N}$ to be a fixed point of $p^{*}$.

There are certainly some similarities between Cohen forcing, in the context of adding a real, and $\mathbb{B}$, in the context of adding a club of $\omega_{1}$ not including any club from the ground model. To point out some obvious examples, both forcing notions add the relevant new object by finite approximations, both are homogeneous (in the above sense) and of the least possible size, both have a simple definition, ${ }^{1}$ and in fact both are absolute in a strong sense (in the sense that any two transitive models of ZF have the same Cohen forcing, and have the same $\mathbb{B}$ in case they agree on $\left.\omega_{1}\right)$. It is therefore natural to ask if there is version of $\operatorname{Add}(\omega, X)$ for $\mathbb{B}$. The main purpose of this note is to present such a forcing. More precisely, I will show that, given a set $X$ of ordinals, there is a forcing, which I will denote by $\operatorname{Add}_{\mathbb{B}}(X)$, which is quite simple to define and which has the following properties.

(1) For every $\operatorname{Add}_{\mathbb{B}}(X)$-generic $G$ and every $\alpha \in X$ one can naturally extract a Baumgartner club $C_{\alpha}^{G}$ from $G$. Furthermore, $C_{\alpha}^{G} \neq C_{\alpha^{\prime}}^{G}$ for all distinct $\alpha, \alpha^{\prime}$ in $X$.

(2) $\operatorname{Add}_{\mathbb{B}}(X)$ is proper and has the $\aleph_{2}-$ c.c.

(3) $\operatorname{Add}_{\mathbb{B}}(X)$ is homogeneous.

(4) For every partition $\left(X_{0}, X_{1}\right)$ of $X, \operatorname{Add}_{\mathbb{B}}(X)$ can be naturally represented as the product $\operatorname{Add}_{\mathbb{B}}\left(X_{0}\right) \times \operatorname{Add}_{\mathbb{B}}\left(X_{1}\right)$. In particular, if $G$ is as in (1) and $\alpha \neq \alpha^{\prime}$ are in $X$, then $C_{\alpha^{\prime}}^{G}$ is $\mathbb{B}$-generic over $\mathbf{V}\left[C_{\alpha}^{G}\right]$.

In the next section I will discuss the forcing axiom for $\mathbb{B}$ for collections of $\lambda$ many dense sets (for arbitrary $\lambda$ ), its consistency and, in Sect. 2.1, its consequences at the level of club guessing at $\omega_{1}$. Subsection 2.2 relates this forcing axiom to the covering number of the meagre ideal $\mathcal{M}_{\mathcal{C}_{\omega_{1}}}$ for the set of all clubs on $\omega_{1}$ endowed with a natural topology. Section 3 introduces the forcing $\operatorname{Add}_{\mathbb{B}}(X)$ and presents its

\footnotetext{
1 For a way to make this precise see for example Zapletal's result on forcings of size $\omega_{1}$ in $L[x], x$ a real, cited right after Definition 2.1 .
} 
basic theory. Finally, Sect. 3.1 presents the main new consistency result in the note, which is the joint consistency of $\mathfrak{b}\left(\omega_{1}\right)=\operatorname{non}\left(\mathcal{M}_{\mathcal{C}_{\omega_{1}}}\right)=\aleph_{2}$ with $\mathfrak{d}\left(\omega_{1}\right)$ large and with very strong failures of club guessing at $\omega_{1}$; these conclusions hold after forcing with $\operatorname{Add}_{\mathbb{B}}(\theta)$ for large $\theta$.

\section{$2 \mathrm{FA}(\mathbb{B})_{\lambda}$, for arbitrary $\lambda$, and strong failures of club guessing}

Given a partial order $\mathcal{P}$ and a cardinal $\lambda$, the forcing axiom for $\mathcal{P}$ and for $\lambda$-many dense sets, denoted by $\operatorname{FA}(\mathcal{P})_{\lambda}$, is the statement that for every $\left\{D_{i} \mid i<\lambda\right\}$, if each $D_{i}$ is a dense subset of $\mathcal{P}$, then there is a filter $G \subseteq \mathcal{P}$ such that $G \cap D_{i} \neq \emptyset$ for all $i$. Also, given a class $\Gamma$ of partial orders, $\operatorname{FA}(\Gamma)_{\lambda}$ means $\operatorname{FA}(\mathcal{P})_{\lambda}$ for all $\mathcal{P} \in \Gamma$. Since Cohen forcing is c.c.c., the forcing axiom for it and for $\lambda$-many dense sets follows from the (consistent) axiom $\mathrm{MA}_{\lambda}$, and the same is of course true for $\operatorname{Add}(\omega, X)$. For the same reason, the forcing axiom PFA, i.e., $\operatorname{FA}(\{\mathcal{P} \mid \mathcal{P} \text { proper }\})_{\aleph_{1}}$-and in fact FA $\left(\left\{\mathcal{P} \mid \mathcal{P} \text { a proper poset of size } \aleph_{1}\right\}\right)_{\aleph_{1}}$, which, unlike PFA, can be forced over any ZFC model -, implies FA $(\mathbb{B})_{\aleph_{1}}$.

A natural question arises at this point: $\operatorname{FA}(\{\mathcal{P} \mid \mathcal{P} \text { proper }\})_{\aleph_{2}}$ is false. But is $\mathrm{FA}(\mathbb{B})_{\aleph_{2}}$ consistent? Note that we cannot use countable-support proper iterated forcing to answer this question as every such forcing will produce a model of $2^{\aleph_{0}} \leq \aleph_{2}$ and $\mathrm{FA}(\mathbb{B})_{\aleph_{2}}$ clearly implies $2^{\aleph_{0}} \geq \aleph_{3}$.

The method of finite-support iterated forcing with symmetric systems of structures as side conditions (see [2] and [3]) can be used to answer this question. This method enables one to force without collapsing cardinals in such a way that the resulting model satisfies $\operatorname{FA}(\mathcal{P})_{\lambda}$, for an arbitrarily fixed $\lambda$, for various well-behaved proper partial orders $\mathcal{P}$ with the $\aleph_{2}-$ c.c. Background information on this method can be found in [2] and [3], so I will not say anything about it here. It turns out that $\mathbb{B}$ is such a well-behaved forcing. More specifically, there is a class of proper posets, which in [3] we refer to as the posets having the $\aleph_{1.5}-$ c.c., to which $\mathbb{B}$ belongs, and such that the forcing axiom $\operatorname{MA}\left(\left\{\mathcal{P} \mid \mathcal{P} \text { has the } \aleph_{1.5}-\text { c.c. }\right\}\right)_{\lambda}-$ which in [3] we call $\mathrm{MA}_{\lambda}^{1.5}$ is consistent for arbitrarily chosen $\lambda$ (cf. Theorem 3.11). The main reason for this terminology is that every c.c.c. poset is in our class and every poset in our class is $\aleph_{2}-$ c.c. It follows that, for any $\lambda, \mathrm{MA}_{\lambda}^{1.5}$ implies both $\mathrm{MA}_{\lambda}$ and $\mathrm{FA}(\mathbb{B})_{\lambda}$. The definition of the $\aleph_{1.5}-$ c.c. is the following.

Definition 2.1 ([3]) A poset $\mathcal{P}$ has the $\aleph_{1.5}-$ c.c. if and only if for every regular cardinal $\theta$ such that $\mathcal{P} \in H(\theta)$ there is a club $D \subseteq[H(\theta)]^{\aleph_{0}}$ such that for every finite $\mathcal{N} \subseteq D$ and every $p \in \mathcal{P}$, if $p \in N$ for some $N \in \mathcal{N}$ such that $\delta_{N}=\min \left\{\delta_{M} \mid M \in \mathcal{N}\right\}$, then there is some condition extending $p$ and $(N, \mathcal{P})$-generic for all $N \in \mathcal{N}$.

Even if $\mathrm{FA}(\mathbb{B})_{\lambda}$ is consistent, for arbitrary $\lambda$, it is not known whether $\operatorname{FA}(\{\mathcal{P} \mid$ $\mathcal{P}$ a proper poset of size $\left.\left.\aleph_{1}\right\}\right)_{\aleph_{2}}$ is consistent.

$\mathbb{B}$ is a very prominent poset with the $\aleph_{1.5}-$ c.c., at least in the sense that it is the simplest such poset which does not have the c.c.c. One way to make mathematical sense of this assertion is to quote the following fact due to Zapletal: If $x \in \mathbb{R}, x^{\sharp}$ exists, and $\mathcal{P} \in L[x]$ is a non-atomic partial order on $\omega_{1}^{\mathbf{V}}$, then $\mathcal{P}$ is forcing-equivalent to the disjoint sum of some number of copies of forcings in 
$\left\{2^{<\omega}, \operatorname{Add}\left(\omega, \omega_{1}\right), \mathbb{B}, \operatorname{Coll}\left(\omega, \omega_{1}\right)\right\}$, where $\operatorname{Coll}\left(\omega, \omega_{1}\right)$ is the collapse of $\omega_{1}$ to $\omega$ with finite conditions ([17]). It is also worth pointing out that, on the other hand, $\mathbb{B}$ is nowhere c.c.c. (i.e., it is not c.c.c. below any condition) and, as Zapletal proved in [18], under PFA it is a minimal nowhere c.c.c. poset, in the sense that every nowhere c.c.c. poset adds a generic for $\mathbb{B}$. Also, if $P=\left\{p_{\alpha} \mid \alpha<\omega_{1}\right\}$ is a proper nowhere c.c.c. forcing notion adding a club $C \subseteq \omega_{1}$ such that for all $\alpha \in C, \dot{G} \cap\left\{p_{\beta} \mid \beta<\alpha\right\}$ is generic for $\left\{p_{\beta} \mid \beta<\alpha\right\}$ (where $\dot{G}$ denotes the generic filter), then $\operatorname{RO}(P)=\operatorname{RO}(\mathbb{B})$ ([16]).

\subsection{Some weakenings of club guessing at $\omega_{1}$}

Club-guessing principles are well studied weakenings of Jensen's diamond principle $\diamond_{\kappa}$, for a cardinal $\kappa$, in which the guessing device is a club-sequence $\left(C_{\delta} \mid \delta \in S\right)$ for some $S \subseteq \kappa \cap \operatorname{Lim}$ (i.e., every $C_{\delta}$ is a club of $\delta$ ) and in which the relevant guessing applies to clubs of $\kappa$ rather than arbitrary subsets of $\kappa$. Unlike $\diamond_{\kappa}$, they are consistent with $2^{\mu}$ large for any given $\mu<\kappa$, simply because they are preserved by $\kappa$-c.c. forcing (since every club of $\kappa$ in any extension by a $\kappa$-c.c. forcing includes a club from the ground model). This is of course the reason why $\mathrm{MA}_{\lambda}$, for any cardinal $\lambda$, is consistent with such guessing principles. Also, whereas club-guessing principles for $\omega_{2}$ or higher regular cardinals are often outright true in ZFC (cf. [14], III, §2), the truth value of their versions at $\omega_{1}$ can be easily changed by forcing (see e.g. $[7,8,15]$ ). Let us see a couple of examples:

A $C$-sequence-also known as a ladder system-is a sequence $\left(C_{\delta} \mid \delta \in \operatorname{Lim}\left(\omega_{1}\right)\right)$ such that $C_{\delta}$ is a cofinal subset of $\delta$ of order type $\omega$ for every $\delta$. Club Guessing at $\omega_{1}$ (CG) says that there is a $C$-sequence $\left(C_{\delta} \mid \delta \in \operatorname{Lim}\left(\omega_{1}\right)\right)$ which guesses clubs in the sense that for every club $D \subseteq \omega_{1}$ there is some $\delta \in \operatorname{Lim}\left(\omega_{1}\right)$ with $C_{\delta} \backslash D$ finite. Kunen's Axiom (KA), also known as Interval Hitting Principle (see e.g. [7]; see also [11]), is the following statement, first considered by Kunen: There is a $C$-sequence $\left(C_{\delta} \mid \delta \in \operatorname{Lim}\left(\omega_{1}\right)\right)$ with the property that for every club $D \subseteq \omega_{1}$ there is some $\delta$ such that $\left[C_{\delta}(n), C_{\delta}(n+1)\right) \cap D \neq \varnothing$ for a tail of $n<\omega$ (where $X(\xi)$ denotes the $\xi$-th member of $X$ if $X$ is a set of ordinals). CG clearly implies KA, follows from $\diamond$, and is preserved by c.c.c. forcing. On the other hand, it is easy to see and a well-known fact that $\mathrm{FA}\left(\left\{\mathcal{P} \mid \mathcal{P} \text { a proper poset of size } \aleph_{1}\right\}\right)_{\aleph_{1}}$ implies $\neg \mathrm{KA}$.

Consider the following weak versions of $\mathrm{CG}$ and $\mathrm{KA}$, respectively: Given a cardinal $\lambda$, let $\mathrm{CG}_{\lambda}$ be the assertion that there is a set $\mathcal{C}$ of subsets of $\omega_{1}$ of order type $\omega$ such that $|\mathcal{C}| \leq \lambda$ and such that for every club $D \subseteq \omega_{1}$ there is some $X \in \mathcal{C}$ such that $X \backslash D$ is finite. Let also $\mathrm{KA}_{\lambda}$ be the assertion that there is a set $\mathcal{C}$ of subsets of $\omega_{1}$ of order type $\omega$ such that $|\mathcal{C}| \leq \lambda$ and such that for every club $D \subseteq \omega_{1}$ there is some $X \in \mathcal{C}$ such that $[X(n), X(n+1)) \cap D \neq \varnothing$ for a tail of $n<\omega$. By a straightforward density argument, if $C$ is $\mathbb{B}$-generic and $X \in \mathbf{V}$ is a subset of $\omega_{1}$ of order type $\omega$, then $[X(n), X(n+1)) \cap C$ is empty for infinitely many $n<\omega$ (see [4]), which immediately implies the following proposition.

Proposition 2.2 For every cardinal $\lambda \geq \omega_{1}$, FA(BB $)_{\lambda}$ implies $\neg \mathrm{KA}_{\lambda}$.

It is worth pointing out that the stronger forcing axiom $\mathrm{MA}_{\lambda}^{1.5}$ implies even stronger failures of club guessing ([3]). 


\section{2 $\mathrm{FA}(\mathbb{B})_{\lambda}$ and the covering number of the meagre ideal of $\mathcal{C}_{\omega_{1}}$}

Recall that for an ideal $\mathcal{I}$ on a set of $X$, the covering number of $\mathcal{I}$, denoted by $\operatorname{cov}(\mathcal{I})$, is the minimal size of a collection of members of $\mathcal{I}$ whose union is $X$. Given a topological space $X$ and a cardinal $\kappa$, let $\mathcal{M}_{X}^{\kappa}$ denote the (possibly improper) ideal on $X \kappa$-generated by the nowhere dense sets, i.e., the collection of all unions of less than $\kappa$-many nowhere dense sets. Let us refer to the members of $\mathcal{M}_{X}^{\kappa}$ as $\kappa$-meagre subsets of $X$. This way, the classical meagre ideal on $X$ is $\mathcal{M}_{X}^{\omega_{1}}$. I will also denote it by $\mathcal{M}_{X}$. The following is a straightforward but useful observation in this general context.

Fact 2.3 $\mathcal{M}_{X}^{\kappa} \subseteq \mathcal{M}_{X}^{\kappa^{\prime}}$ and therefore $\operatorname{cov}\left(\mathcal{M}_{X}^{\kappa^{\prime}}\right) \leq \operatorname{cov}\left(\mathcal{M}_{X}^{\kappa}\right)$ whenever $\kappa \leq \kappa^{\prime}$, and $\operatorname{cov}\left(\mathcal{M}_{X}^{\omega}\right)=\operatorname{cov}\left(\mathcal{M}_{X}^{\kappa}\right)$ for every $\kappa<\operatorname{cov}\left(\mathcal{M}_{X}^{\omega}\right)$.

In the following definition and throughout the note, if $C$ is a club of $\omega_{1}, \tilde{C}$ will denote its enumerating function.

Definition 2.4 Consider the topology $\tau_{\mathbb{B}}$ on the set of all clubs of $\omega_{1}$ whose basis is given by the conditions in $\mathbb{B}$; in other words, a basis for this topology is given by $\left\{B_{p} \mid p \in \mathbb{B}\right\}$, where $B_{p}$ is the set of all clubs $C \subseteq \omega_{1}$ such that $p \subseteq \tilde{C}$. Then $\mathcal{C}_{\omega_{1}}$ denotes the set of all clubs of $\omega_{1}$ endowed with the topology $\tau_{\mathbb{B}}$.

One first observation is that the usual Baire category theorem holds for $\mathcal{C}_{\omega_{1}}$ :

Lemma $2.5 \operatorname{cov}\left(\mathcal{M}_{\mathcal{C}_{\omega_{1}}}\right) \geq \aleph_{1}$

Proof Let $\left(D_{n}\right)_{n<\omega}$ be a sequence of dense subsets of $\mathbb{B}$. It suffices to show that there is a club $C \subseteq \omega_{1}$ such that for all $n, p \subseteq \tilde{C}$ for some $p \in D_{n}$.

Let $\left(C_{\delta}: \delta \in \operatorname{Lim}\left(\omega_{1}\right)\right)$ be a $C$-sequence. For each $n$, let $D_{n}^{*}$ denote the set of $\mathbb{B}$-conditions $p$ extending some condition in $D_{n}$ and such that for every limit ordinal $\delta \in \operatorname{dom}(p)$ there is some $\delta^{\prime} \in \operatorname{dom}(p) \cap \delta$ such that $p\left(\delta^{\prime}\right)>C_{p(\delta)}(n)$, where $\left(C_{p(\delta)}(i)\right)_{i<\omega}$ is the increasing enumeration of $C_{p(\delta)}$. Each $D_{n}^{*}$ is clearly dense in $\mathbb{B}$. Let $N$ be a countable elementary substructure of $H\left(\omega_{2}\right)$ containing each $D_{n}^{*}$ and let $\left(\delta_{n}\right)_{n<\omega}$ be an enumeration of $\delta_{N}$. Now we build a decreasing sequence $\left(p_{n}\right)_{n<\omega}$ of $\mathbb{B}$-conditions such that for each $n, p_{n} \in N \cap D_{n}^{*}$ and $\delta_{n} \in \operatorname{dom}\left(p_{n}\right)$. This is possible since each $D_{n}^{*}$ is dense. In the end, $p^{*}=\bigcup_{n} p_{n}: \delta_{N} \longrightarrow \delta_{N}$ is a strictly increasing and continuous function by the choice of $\left(D_{n}^{*}\right)_{n<\omega}$ and of course range $\left(p^{*}\right)$ is cofinal in $\delta_{N}$. Hence range $\left(p^{*}\right) \cup\left(\omega_{1} \backslash \delta_{N}\right)$ is a club as desired.

Given a partial order $\mathcal{P}$, let $\mathfrak{m}(\mathcal{P})$ be the minimal size of a family $\mathcal{D}$ of dense subsets of $\mathcal{P}$ such that there is no filter $G \subseteq \mathcal{P}$ intersecting all members of $\mathcal{D}$ (i.e., $\mathfrak{m}(\mathcal{P})$ is the least cardinal $\lambda$ such that $\operatorname{FA}(\mathcal{P})_{\lambda}$ fails). If $\mathcal{P}$ is infinite, $\mathfrak{m}(\mathcal{P})$ is of course at least $\aleph_{1}$.

Proposition 2.6 $\operatorname{cov}\left(\mathcal{M}_{\mathcal{C}_{\omega_{1}}}\right)=\mathfrak{m}(\mathbb{B})$

Proof The proof is a standard translation exercise between topological notions and order-theoretical notions, and is essentially identical to the proof that $\mathfrak{m}$ (Cohen) is the covering number of the meagre ideal for the Baire space (see for example [10], Theorem 16.1). It is easy to see that $\operatorname{cov}\left(\mathcal{M}_{\mathcal{C}_{\omega_{1}}}\right) \leq \mathfrak{m}(\mathbb{B})$. In fact, if $\mathcal{D}$ is a collection 
of dense subsets of $\mathbb{B}$ and there is no filter of $\mathbb{B}$ intersecting all members of $\mathcal{D}$, then $\left\{X_{D} \mid D \in \mathcal{D}\right\}$ is a collection of nowhere dense sets covering $\mathcal{C}_{\omega_{1}}$, where $X_{D}$ is, for every $D \in \mathcal{D}$, the collection of $C \in \mathcal{C}_{\omega_{1}}$ such that $p \nsubseteq \tilde{C}$ for any $p \in D$. Hence, in order to prove the equality we may assume, by the previous lemma, that $\mathfrak{m}(\mathbb{B})>\aleph_{1}$. Now, given $\lambda<\mathfrak{m}(\mathbb{B})$ and a collection $\left\{X_{i} \mid i<\lambda\right\}$ of closed nowhere dense subsets of $\mathcal{C}_{\omega_{1}}, D_{i}=\left\{p \in \mathbb{B} \mid B_{p} \cap X_{i}=\emptyset\right\}$ is a dense open subset of $\mathbb{B}$ for all $i$. Let $\left\{E_{v} \mid v<\omega_{1}\right\}$ be a set of dense subsets of $\mathbb{B}$ such that $\bigcup \operatorname{range}(G)$ is a club of $\omega_{1}$ for every filter $G \subseteq \mathbb{B}$ meeting all $E_{\nu}$. Since $\mathfrak{m}(\mathbb{B})>\aleph_{1}$, we can find a filter $G \subseteq \mathbb{B}$ meeting all $E_{v}$ and all $D_{i}$. It follows then that $C=\bigcup \operatorname{range}(G)$ is a club of $\omega_{1}$ such that $C \notin X_{i}$ of all $i$.

Of course there is nothing special about Cohen forcing or $\mathbb{B}$ in any of these translations; in fact, a similar characterisation can be always obtained for $\mathfrak{m}(\mathcal{P})$ for any poset $\mathcal{P}$. What is nice about Cohen forcing and $\mathbb{B}$ is the appealing appearance of the topological side of the translation.

\section{Adding many Baumgartner clubs}

The following proposition shows that some nontrivial move is necessary in order to add many Baumgartner clubs by a product forcing not collapsing $\omega_{1}$.

Proposition 3.1 Both the finite support product of $\aleph_{0}$ copies of $\mathbb{B}$ and the countable support product of $\aleph_{0}$ copies of $\mathbb{B}$ collapse $\omega_{1}$.

Proof Let $\mathcal{P}_{0}$ be the finite support product of copies of $\mathbb{B}$ indexed by $\omega$ and let $\mathcal{P}_{1}$ be the countable support product of copies of $\mathbb{B}$ indexed by $\omega$. The first part is trivial: Given any $f \in \mathcal{P}_{0}$ and any $\alpha<\omega_{1}$ we can find an extension $f^{\prime}$ of $f$ and some $n \in \operatorname{dom}\left(f^{\prime}\right)$ such that $f^{\prime}(n)(0)>\alpha$. This gives a cofinal function $\dot{f}: \omega \longrightarrow \omega_{1}^{\mathbf{V}}$ in the extension by $\mathcal{P}_{0}$.

The second part can be argued for by a variation of a standard argument for showing the well-known fact that the full support product of countably many copies of Cohen forcing collapses $\mathbb{R}^{\mathbf{V}}$ to $\omega$ (see for example [6]): We start out by fixing a ladder system $\left(C_{\delta} \mid \delta \in \operatorname{Lim}\left(\omega_{1}\right)\right)$. Given a $\mathcal{P}_{1}$-generic filter $G$ and $n<\omega$ let $F^{n}: \omega_{1}^{\mathbf{V}} \longrightarrow \omega_{1}^{\mathbf{V}}$ be the strictly increasing and continuous function added by $G$ on the $n$-th coordinate and let $\delta_{n}=F^{n}(\omega) \in \operatorname{Lim}\left(\omega_{1}\right)$. Now, given any $k<\omega$, look at the biggest $n_{0}<\omega$ such that the interval $\left[F^{0}(k+i), F^{0}(k+i+1)\right)$ contains exactly one element of $C_{\delta_{0}}$ for every $i<n_{0}$, which exists by density. Now write $a_{0}=0$ or $a_{0}=1$ depending on whether or not $\left|\left[F^{1}\left(n_{0}\right), F^{1}\left(n_{0}+1\right)\right) \cap C_{\delta_{1}}\right|=1$. Let $n_{1}<\omega$ be biggest such that $\left|\left[F^{1}\left(n_{0}+1+i\right), F^{1}\left(n_{0}+2+i\right)\right) \cap C_{\delta_{1}}\right|=1$ for all $i<n_{1}$, write $a_{1}=0$ or $a_{1}=1$ depending on whether or not $\left|\left[F^{2}\left(n_{1}\right), F^{2}\left(n_{1}+1\right)\right) \cap C_{\delta_{2}}\right|=1$, and keep going. This way we associate to $k$ a unique $\left(a_{n}\right)_{n<\omega} \in{ }^{\omega} 2$, and it is easy to check that, by density, every real in $\mathbf{V}$ is the image of some $k<\omega$ under this mapping.

The following notation will be used in the remainder of this section. Given two functions $\mathcal{F}$ and $\mathcal{G}$, I will denote by $\mathcal{F} \oplus \mathcal{G}$ the function $\mathcal{H}$ with $\operatorname{domain} \operatorname{dom}(\mathcal{F}) \cup$ $\operatorname{dom}(\mathcal{G})$ such that 
- $\mathcal{H}(x)=\mathcal{F}(x)$ for every $x \in \operatorname{dom}(\mathcal{F}) \backslash \operatorname{dom}(\mathcal{G})$,

- $\mathcal{H}(x)=\mathcal{G}(x)$ for every $x \in \operatorname{dom}(\mathcal{G}) \backslash \operatorname{dom}(\mathcal{F})$, and

- $\mathcal{H}(x)=\mathcal{F}(x) \cup \mathcal{G}(x)$ for every $x \in \operatorname{dom}(\mathcal{F}) \cap \operatorname{dom}(\mathcal{G})$.

Note that $(\mathcal{F} \oplus \mathcal{G}) \oplus \mathcal{H}=\mathcal{F} \oplus(\mathcal{G} \oplus \mathcal{H})$ for all functions $\mathcal{F}, \mathcal{G}$ and $\mathcal{H}$. I will denote $(\mathcal{F} \oplus \mathcal{G}) \oplus \mathcal{H}$ simply by $\mathcal{F} \oplus \mathcal{G} \oplus \mathcal{H}$.

The following simple fact will be used repeatedly.

Fact $3.2 \operatorname{ot}(A \cup B)<\delta$ whenever $\delta$ is an indecomposable ordinal and $A, B$ are sets of ordinals with ot $(A), \operatorname{ot}(B)<\delta$.

Proof Since ot $(A \cup B) \leq \operatorname{ot}(A)+\operatorname{ot}(B)$.

I will now define $\operatorname{Add}_{\mathbb{B}}(X) . \operatorname{Add}_{\mathbb{B}}(X)$ can be seen as a forcing adding Baumgartner clubs, indexed by ordinals in $X$, by using finite supports and with countable subsets of $X$ as side conditions. The definition of $\operatorname{Add}_{\mathbb{B}}(X)$ is a streamlined version of the constructions from [2] or [3]. In fact, it is simple enough that it is an actual product (Lemma 3.5), whereas the constructions in [2] and [3] certainly are not. The fact that $\operatorname{Add}_{\mathbb{B}}(X)$ is a product is crucial for the proof (in Theorem 3.15) that there is a Luzin set of clubs of size $\aleph_{2}$ in the extension.

Definition 3.3 Let $X$ be a set of ordinals. $\operatorname{Add}_{\mathbb{B}}(X)$ is the following forcing notion: Conditions in $\operatorname{Add}_{\mathbb{B}}(X)$ are pairs of the form $p=(f, \mathcal{F})$ with the following properties.

(1) $f$ is a finite function with $\operatorname{dom}(f) \subseteq X$ and such that $f(\alpha) \in \mathbb{B}$ for every $\alpha \in \operatorname{dom}(f)$.

(2) $\mathcal{F}$ is a finite function with $\operatorname{dom}(\mathcal{F}) \subseteq \omega_{1}$ such that for every $\delta \in \operatorname{dom}(\mathcal{F})$,

(a) $\delta$ is a countable indecomposable ordinal,

(b) $\mathcal{F}(\delta)$ is a countable subset of $X$,

(c) $\delta \in \operatorname{dom}(f(\alpha))$ and $f(\alpha)(\delta)=\delta$ for all $\alpha \in \operatorname{dom}(f) \cap \mathcal{F}(\delta)$, and

(d) $\operatorname{ot}\left(\mathcal{F}\left(\delta^{\prime}\right)\right)<\delta$ for every $\delta^{\prime} \in \operatorname{dom}(\mathcal{F}\lceil\delta)$.

Given $\operatorname{Add}_{\mathbb{B}}(X)$ conditions $\left(f_{0}, \mathcal{F}_{0}\right),\left(f_{1}, \mathcal{F}_{1}\right),\left(f_{1}, \mathcal{F}_{1}\right)$ extends $\left(f_{0}, \mathcal{F}_{0}\right)$ iff

- $\operatorname{dom}\left(f_{0}\right) \subseteq \operatorname{dom}\left(f_{1}\right)$ and $f_{0}(\alpha) \subseteq f_{1}(\alpha)$ for every $\alpha \in \operatorname{dom}\left(f_{0}\right)$, and

- $\operatorname{dom}\left(\mathcal{F}_{0}\right) \subseteq \operatorname{dom}\left(\mathcal{F}_{1}\right)$ and $\mathcal{F}_{0}(\delta) \subseteq \mathcal{F}_{1}(\delta)$ for every $\delta \in \operatorname{dom}\left(\mathcal{F}_{0}\right)$.

Let us fix a set $X$ of ordinals and let us prove the relevant facts about $\operatorname{Add}_{\mathbb{B}}(X)$.

Given $\alpha \in X$ and a generic filter $G$ for $\operatorname{Add}_{\mathbb{B}}(X)$, let $F_{G}^{\alpha}=\{f(\alpha) \mid(f, \mathcal{F}) \in$ $G$ for some $\mathcal{F}$ \}. The following fact is clear.

Fact 3.4 Given $\alpha \in X$ and a generic filter $G$ for $\operatorname{Add}_{\mathbb{B}}(X), F_{G}^{\alpha}$ is a generic filter for $\mathbb{B}$.

Given a condition $p=(f, \mathcal{F})$ in $\operatorname{Add}_{\mathbb{B}}(X)$ and $Y \subseteq X$, let $p \mid Y=$ $(f|Y, d, N \cap Y\rangle \mid\langle\delta, N\rangle \in \mathcal{F}, \delta \in \operatorname{dom}(\mathcal{F})\})$.

Lemma 3.5 follows from Fact 3.2.

Lemma 3.5 Let $X_{0}, X_{1}$ be disjoint sets of ordinals. Then, the function sending a pair $\left(\left(f_{0}, \mathcal{F}_{0}\right),\left(f_{1}, \mathcal{F}_{1}\right)\right) \in \operatorname{Add}_{\mathbb{B}}\left(X_{0}\right) \times \operatorname{Add}_{\mathbb{B}}\left(X_{1}\right)$ to $\left(f_{0} \cup f_{1}, \mathcal{F}_{0} \oplus \mathcal{F}_{1}\right)$ is an isomorphism between the posets $\operatorname{Add}_{\mathbb{B}}\left(X_{0}\right) \times \operatorname{Add}_{\mathbb{B}}\left(X_{1}\right)$ and $\operatorname{Add}_{\mathbb{B}}\left(X_{0} \cup X_{1}\right)$. The inverse of this function is the function sending $p \in \operatorname{Add}_{\mathbb{B}}(X)$ to $\left(p\left\lceil X_{0}, p\left\lceil X_{1}\right)\right.\right.$. 
Lemma 3.6 $\operatorname{Add}_{\mathbb{B}}(X)$ has the $\aleph_{2}-$ c.c.

Proof Given $\operatorname{Add}_{\mathbb{B}}(X)$ conditions $\left(f_{\xi}, \mathcal{F}_{\xi}\right)$ for $\xi<\omega_{2}$, we may assume that $\left\{\operatorname{dom}\left(f_{\xi}\right) \mid \xi<\omega_{2}\right\}$ forms a $\Delta$-system with root $R$ and that $f_{\xi}(\alpha)=f_{\xi^{\prime}}(\alpha)$ for all $\xi, \xi^{\prime}<\omega_{2}$ and all $\alpha \in R$. We may also assume that $\operatorname{dom}\left(\mathcal{F}_{\xi}\right)=\operatorname{dom}\left(\mathcal{F}_{\xi^{\prime}}\right)$ for all $\xi, \xi^{\prime}<\omega_{2}$. Since $\left\{\operatorname{dom}\left(f_{\xi}\right) \mid \xi<\omega_{2}\right\}$ forms a $\Delta$-system, there is a club $D \subseteq \omega_{2}$ such that for all $\xi<\xi^{\prime}$ in $D, \bigcup \operatorname{range}\left(\mathcal{F}_{\xi}\right) \cap \operatorname{dom}\left(f_{\xi^{\prime}}\right) \subseteq R$. In fact we may assume that for all $\xi<\xi^{\prime}$ in $D, \bigcup \operatorname{range}\left(\mathcal{F}_{\xi}\right) \cap \operatorname{dom}\left(f_{\xi^{\prime \prime}}\right) \subseteq R$ for every $\xi^{\prime \prime}<\omega_{2}$ such that $\xi^{\prime} \leq \xi^{\prime \prime}$ : Let $\bar{\xi}<\omega_{2}$ and assume $D \cap \bar{\xi}$ has been defined. We may clearly assume that $D \cap \bar{\xi}$ has a maximum, $\xi_{0}$. There must then be a least $\zeta<\omega_{2}, \zeta \geq \bar{\xi}$, such that $\bigcup \operatorname{range}\left(F_{\xi_{0}}\right) \cap \operatorname{dom}\left(f_{\zeta^{\prime}}\right) \subseteq R$ for all $\zeta^{\prime} \geq \zeta$. Otherwise there are $\aleph_{2}$-many $\zeta<\omega_{2}$ such that $\bigcup \operatorname{range}\left(F_{\xi_{0}}\right) \cap \operatorname{dom}\left(f_{\zeta}\right) \nsubseteq R$. But then, since $\left|\bigcup \operatorname{range}\left(F_{\xi_{0}}\right)\right| \leq \aleph_{0}$, there must be $\zeta \neq \zeta^{\prime}$ and some $\alpha \in \operatorname{dom}\left(f_{\zeta}\right) \cap \operatorname{dom}\left(f_{\zeta^{\prime}}\right)$ such that $\alpha \in \bigcup \operatorname{range}\left(F_{\xi_{0}}\right) \backslash R$. This is of course impossible since $\operatorname{dom}\left(f_{\zeta}\right) \cap \operatorname{dom}\left(f_{\zeta^{\prime}}\right)=R$. Now we can of course set such a $\zeta$ to be the least member of $D$ above $\xi_{0}$.

Let $\xi^{*}$ be the $\omega_{1}$-th member of $D$. Since $\bigcup \operatorname{range}\left(\mathcal{F}_{\xi^{*}}\right)$ is countable, again by the fact that $\left\{\operatorname{dom}\left(f_{\xi}\right) \mid \xi<\omega_{1}\right\}$ forms a $\Delta$-system and therefore the sets $\operatorname{dom}\left(f_{\xi}\right) \backslash R$ (for $\left.\xi<\omega_{1}\right)$ are pairwise disjoint, there must be some $\xi^{* *}<\xi^{*}$ such that $\operatorname{dom}\left(f_{\xi^{* *}}\right) \cap \bigcup \operatorname{range}\left(\mathcal{F}_{\xi^{*}}\right) \subseteq R$. Using Fact 3.2 it follows then immediately that $\left(f_{\xi^{* *}} \cup f_{\xi^{*}}, \mathcal{F}_{\xi^{* *}} \oplus \mathcal{F}_{\xi^{*}}\right)$ is a condition in $\operatorname{Add}_{\mathbb{B}}(X)$ and that it extends both $\left(f_{\xi * *}, \mathcal{F}_{\xi^{* *}}\right)$ and $\left(f_{\xi *}, \mathcal{F}_{\xi *}\right)$.

Note that Lemma 3.6 is true in ZFC. This is in contrast, for example, with corresponding lemmas in [2] and [3], for which $\mathrm{CH}$ is needed.

Next comes the properness lemma. As we will see, the proof of the lemma proceeds quite naturally by induction on the initial segments of $X$. Most features of the definition of $\operatorname{Add}_{\mathbb{B}}(X)$ are there precisely to make the proof of Lemma 3.7 go through.

Lemma $3.7 \operatorname{Add}_{\mathbb{B}}(X)$ is proper.

Proof Let $M$ be a countable elementary substructure of any large enough $H(\lambda)$ containing $X$, let $p=(f, \mathcal{F}) \in \operatorname{Add}_{\mathbb{B}}(X) \cap M$, and let $N=M \cap X$. Let $f^{*}$ be the function with the same domain as $f$ such that $f^{*}(\alpha)=f(\alpha) \cup\left\{\left\langle\delta_{M}, \delta_{M}\right\rangle\right\}$ for every $\alpha \in \operatorname{dom}(f)$, and let $p^{*}=\left(f^{*}, \mathcal{F} \cup\left\{\left\langle\delta_{M}, N\right\rangle\right\}\right)$. For every $N^{\prime} \in \mathcal{F}$, since $N^{\prime} \in M$ and $N^{\prime}$ is countable in $M$, ot $\left(N^{\prime}\right)<\delta_{M}$. It follows that $p^{*}$ is a condition in $\operatorname{Add}_{\mathbb{B}}(X)$. Hence, it suffices to show by induction on the ordinals $\gamma$ that if $\gamma \in M$, then $p^{*} \uparrow(X \cap \gamma)$ is $\left(M, \operatorname{Add}_{\mathbb{B}}(X \cap \gamma)\right)$-generic.

For this, we may clearly assume $\gamma>0$. Let $A$ be any maximal antichain of $\operatorname{Add}_{\mathbb{B}}(X \cap \gamma), A \in M$, and let $p^{\prime}=\left(f^{\prime}, \mathcal{F}^{\prime}\right)$ be any condition extending $p^{*} \uparrow(X \cap \gamma)$ and extending some condition $\tilde{p}=(\tilde{f}, \tilde{\mathcal{F}})$ in $A$. It suffices to see that $\tilde{p}$ is compatible with a condition in $M \cap A$ as then it will of course follow that $\tilde{p} \in M$.

Suppose $\gamma=\gamma_{0}+1$. We may assume without loss of generality that $\gamma_{0} \in \operatorname{dom}\left(f^{\prime}\right)$. Let $G$ be $\operatorname{Add}_{\mathbb{B}}\left(X \cap \gamma_{0}\right)$-generic such that $p^{\prime} \uparrow\left(X \cap \gamma_{0}\right) \in G$. In $M[G]$ there is a condition $q=(g, \mathcal{G})$ with the following properties.

(i) $q$ extends a condition in $A$.

(ii) $q \uparrow\left(X \cap \gamma_{0}\right) \in G$. 
(iii) $\gamma_{0} \in \operatorname{dom}(g)$ and $g\left(\gamma_{0}\right)$ end-extends $f^{\prime}\left(\gamma_{0}\right) \uparrow \delta_{M}$ (i.e., $f^{\prime}\left(\gamma_{0}\right) \uparrow \delta_{M} \subseteq g\left(\gamma_{0}\right)$ and, for all $\left.v \in \operatorname{dom}\left(f^{\prime}\left(\gamma_{0}\right) \uparrow \delta_{M}\right), g\left(\gamma_{0}\right) \uparrow v=f^{\prime}\left(\gamma_{0}\right) \uparrow v\right)$.

(iv) $\operatorname{ot}\left(\mathcal{G}\left(\delta^{\prime}\right)\right)<\delta$ for every $\delta \in \operatorname{dom}\left(\mathcal{F}^{\prime}\left\lceil\delta_{M}\right)\right.$ and every $\delta^{\prime} \in \operatorname{dom}(\mathcal{G}\lceil\delta)$.

(v) $\operatorname{ot}\left(\mathcal{F}^{\prime}\left(\delta^{\prime}\right)\right)<\delta$ for every $\delta \in \operatorname{dom}(\mathcal{G})$ and every $\delta^{\prime} \in \operatorname{dom}\left(\mathcal{F}^{\prime} \uparrow \delta\right)$.

The existence of such a $q$ is witnessed by $p^{\prime}$ and can be expressed by a sentence with $G, f^{\prime}\left(\gamma_{0}\right)\left\lceil\delta_{M}\right.$ and $\left\{\left\langle\delta, \operatorname{ot}\left(\mathcal{F}^{\prime}(\delta)\right)\right\rangle \mid \delta \in \operatorname{dom}\left(\mathcal{F}^{\prime}\left\lceil\delta_{M}\right)\right\}\right.$ as parameters. Hence there is such a $q$ in $M[G]$ as these parameters are in $M[G] \preccurlyeq H(\lambda)[G]$. Note that $q \in M$ since $M[G] \cap \mathbf{V}=M$ by the induction hypothesis. Since $p^{\prime} \uparrow\left(X \cap \gamma_{0}\right) \in G$, it follows that $p^{\prime} \uparrow\left(X \cap \gamma_{0}\right)$ and $q \uparrow\left(X \cap \gamma_{0}\right)$ are compatible as $\operatorname{Add}_{\mathbb{B}}\left(X \cap \gamma_{0}\right)$ conditions. Let $r=(h, \mathcal{H})$ be a lower bound for them. Then

$$
\left(h \cup\left\{\left\langle\gamma_{0}, f^{\prime}\left(\gamma_{0}\right) \cup g\left(\gamma_{0}\right)\right\rangle\right\}, \mathcal{H} \oplus \mathcal{F}^{\prime} \oplus \mathcal{G}\right)
$$

is a common extension of $p^{\prime}$ and $q$ using Fact 3.2.

Next suppose $\gamma$ is a nonzero limit ordinal with $\operatorname{cf}(\gamma) \neq \omega_{1}$. By either the fact that $Z \subseteq M$ for some cofinal subset $Z$ of $\gamma$-when $\operatorname{cf}(\gamma)=\omega$-or the fact that there is some $\sigma_{0} \in M$ bounding $\operatorname{dom}\left(h\lceil\gamma)\right.$ for every $(h, \mathcal{H}) \in A-$ when $\operatorname{cf}(\gamma) \geq \omega_{2}$, as $|A| \leq \aleph_{1}$ by Lemma 3.6, there is some $\sigma \in M \cap \gamma$ bounding $\operatorname{dom}(\tilde{f} \uparrow \gamma)$. Let $G$ be $\operatorname{Add}_{\mathbb{B}}(X \cap \sigma)$-generic such that $p^{\prime} \uparrow(X \cap \sigma) \in G$. In $M[G]$ we may then find some $q=(g, \mathcal{G})$ in $A$ with the following properties.

(i) $q \uparrow(X \cap \sigma) \in G$.

(ii) $\operatorname{dom}(g) \subseteq \sigma$.

(iii) $\operatorname{ot}\left(\mathcal{G}\left(\delta^{\prime}\right)\right)<\delta$ for every $\delta \in \operatorname{dom}\left(\mathcal{F}^{\prime} \uparrow \delta_{M}\right)$ and every $\delta^{\prime} \in \operatorname{dom}(\mathcal{G} \uparrow \delta)$.

(iv) $\operatorname{ot}\left(\mathcal{F}^{\prime}\left(\delta^{\prime}\right)\right)<\delta$ for every $\delta \in \operatorname{dom}(\mathcal{G})$ and every $\delta^{\prime} \in \operatorname{dom}\left(\mathcal{F}^{\prime} \uparrow \delta\right)$.

As in the previous case, the existence of such a $q$ is witnessed by $\tilde{p}$ and can be expressed by a sentence with parameters in $M[G]$. Also as in the previous case, using that $M[G] \cap \mathbf{V}=M$, which is true by the induction hypothesis, we may assume that $q$ is in fact in $M$. Again, we may find a common extension $(h, \mathcal{H})$ of $\tilde{p} \uparrow(X \cap \sigma)$ of $q \uparrow(X \cap \sigma)$. But then, $\left(h, \mathcal{H} \oplus \mathcal{G} \oplus \mathcal{F}^{\prime}\right)$ is a common extension of $\tilde{p}$ and $q$. Note that in the case $\operatorname{cf}(\gamma) \geq \omega_{2}$ the proof does not produce a common extension of $p^{\prime}$ and of some condition in $A \cap M$, but only of $\tilde{p}$ and some condition in $A \cap M$. This is of course enough for our purposes.

Finally suppose $\gamma$ is a nonzero limit ordinal with $\operatorname{cf}(\gamma)=\omega_{1}$. Let $\sigma \in \gamma \cap M$ be such that $\operatorname{dom}\left(f^{\prime}\lceil\sup (M \cap \gamma))<\sigma\right.$ and let $\left(\gamma_{\nu}\right)_{\nu<\omega_{1}} \in M$ be a strictly increasing and continuous sequence of ordinals converging to $\gamma$. Let $D$ be the set of all conditions in $\operatorname{Add}_{\mathbb{B}}(X \cap \gamma)$ extending some condition in $A$. Let $G$ be $\operatorname{Add}_{\mathbb{B}}(X \cap \sigma)$-generic with $p^{\prime} \uparrow(X \cap \sigma) \in G$, and let $C \in M[G]$ be the club of $v \in \omega_{1}=\omega_{1}^{V[G]}$-where the equality holds by the induction hypothesis—-such that for every $v^{\prime}<v$ there is some $q=(g, \mathcal{G}) \in D$ such that

(i) $q \uparrow(X \cap \sigma) \in G$,

(ii) $\operatorname{dom}(g) \backslash \sigma \subseteq\left[\gamma_{v^{\prime}}, \gamma_{v}\right)$,

(iii) $\operatorname{ot}\left(\mathcal{G}\left(\delta^{\prime}\right)\right)<\delta$ for every $\delta \in \operatorname{dom}\left(\mathcal{F}^{\prime} \uparrow \delta_{M}\right)$ and every $\delta^{\prime} \in \operatorname{dom}(\mathcal{G}\lceil\delta)$, and

(iv) $\operatorname{ot}\left(\mathcal{F}^{\prime}\left(\delta^{\prime}\right)\right)<\delta$ for every $\delta \in \operatorname{dom}(\mathcal{G})$ and every $\delta^{\prime} \in \operatorname{dom}\left(\mathcal{F}^{\prime} \uparrow \delta\right)$. 
$C$, being defined with $\left\{\left\langle\delta, \operatorname{ot}\left(\mathcal{F}^{\prime}(\delta)\right)\right\rangle \mid \delta \in \operatorname{dom}\left(\mathcal{F}^{\prime}\left\lceil\delta_{M}\right)\right\}, G \in M[G]\right.$ as parameters, is in $M[G]$, and it is clearly closed by definition.

To see that $C$ is unbounded, note that for every $v<\omega_{1}$ there is some $q=(g, \mathcal{G}) \in$ $D$ such that $\operatorname{dom}(g) \backslash \sigma \subseteq\left[\gamma_{\nu}, \gamma\right), q \uparrow(X \cap \sigma) \in G$, ot $\left(\mathcal{G}\left(\delta^{\prime}\right)\right)<\delta$ for every $\delta \in \operatorname{dom}\left(\mathcal{F}^{\prime}\left\lceil\delta_{M}\right)\right.$ and every $\delta^{\prime} \in \operatorname{dom}\left(\mathcal{G}\lceil\delta)\right.$, and such that $\operatorname{ot}\left(\mathcal{F}^{\prime}\left(\delta^{\prime}\right)\right)<\delta$ for every $\delta \in \operatorname{dom}(\mathcal{G})$ and every $\delta^{\prime} \in \operatorname{dom}\left(\mathcal{F}^{\prime}\lceil\delta)\right.$, as this is witnessed by $p^{\prime}$. Now, since $\operatorname{ot}\left(C \cap \delta_{M}\right)=\delta_{M}$ and ot $\left(\mathcal{F}^{\prime}(\delta)\right)<\delta_{M}$ for every $\delta \in \operatorname{dom}\left(\mathcal{F}^{\prime}\left\lceil\delta_{M}\right)\right.$, using Fact 3.2 we may find some $v \in C \cap \delta_{M}$ and some $v^{\prime}<v$ such that $\left[v^{\prime}, v\right)$ has empty intersection with $\bigcup\left\{\mathcal{F}^{\prime}(\delta) \mid \delta \in \operatorname{dom}\left(\mathcal{F}^{\prime}\left\lceil\delta_{M}\right)\right\}\right.$.

Let $q=(g, \mathcal{G}) \in D \cap M[G]$ be such that (i)-(iv) above hold for $q$ with this particular choice of $v$ and $v^{\prime}$, and note that $q \in M$ again since $M[G] \cap \mathbf{V}=M$ by the induction hypothesis.

Let $\left(\delta_{i}\right)_{i<n}$ be an enumeration of $\operatorname{dom}\left(\mathcal{F}^{\prime}\right) \backslash \delta_{M}$ and let $g^{\prime}$ be the function with domain $\operatorname{dom}(g) \backslash \sigma$ such that $g^{\prime}(\alpha)=g(\alpha) \cup\left\{\left\langle\delta_{i}, \delta_{i}\right\rangle \mid i<n\right\}$ for all $\alpha \in \operatorname{dom}\left(g^{\prime}\right)$. As in the previous two cases we may find a condition $(h, \mathcal{H})$ extending $p^{\prime} \uparrow(X \cap \sigma)$ and $q \uparrow(X \cap \sigma)$. Then $\left(h \cup g^{\prime}, \mathcal{H} \oplus \mathcal{F}^{\prime} \oplus \mathcal{G}\right)$ is a common extension of $p^{\prime}$ and $q$. This completes the proof of the Lemma.

It would be nice to have that $\operatorname{Add}_{\mathbb{B}}(X)$ has the $\aleph_{1.5}-$ c.c. (in the same way that both Cohen forcing and $\operatorname{Add}(\omega, X)$ have the c.c.c.). Unfortunately this does not seem to be the case with the official definition of $\aleph_{1.5}$-c.c. (Definition 2.1). Nevertheless, $\operatorname{Add}_{\mathbb{B}}(X)$ belongs to a slightly bigger class $\Gamma$ such that a model of the corresponding forcing axiom $\mathrm{FA}(\Gamma)_{\lambda}$ for every $\lambda<2^{\aleph_{0}}$ (and $2^{\aleph_{0}}$ arbitrarily large) can be built by the same construction as in [3] (replacing of course everywhere in that construction $\aleph_{1.5}-$ c.c. by the following slightly more general class). The definition of this relaxed $\aleph_{1.5}-$ c.c. is obtained by restricting a bit the collections of finite families $\mathcal{N} \subseteq D$ under consideration. The families we will be considering are what I call here ${ }^{2} \operatorname{Add}_{\mathbb{B}}$-friendly.

Definition 3.8 A set $\mathcal{N}$ of countable sets such that $\delta_{N}$ exists for every $N \in \mathcal{N}$ is $\operatorname{Add}_{\mathbb{B}}$-friendly if and only if for all $N, N^{\prime} \in \mathcal{N}$, if $\delta_{N^{\prime}}<\delta_{N}$, then ot $\left(N^{\prime} \cap \operatorname{Ord}\right)<\delta_{N}$.

The relevant weakening of the $\aleph_{1.5}-$ c.c. is the following.

Definition 3.9 A partial order $\mathcal{P}$ has the $\operatorname{Add}_{\mathbb{B}}-\aleph_{1.5}-$ c.c. if for every regular $\theta$ such that $\mathcal{P} \in H(\theta)$ there is a club $D \subseteq[H(\theta)]^{\aleph_{0}}$ such that for every finite $\operatorname{Add}_{\mathbb{B}}$-friendly $\mathcal{N} \subseteq D$ and every $p \in \mathcal{P}$, if $p \in N$ for some $N \in \mathcal{N}$ such that $\delta_{N}=\min \left\{\delta_{M} \mid M \in\right.$ $\mathcal{N}\}$, then there is an extension of $p$ which is $(N, \mathcal{P})$-generic for every $N \in \mathcal{N}$.

Every poset with the $\aleph_{1.5}-$ c.c. obviously has the $\operatorname{Add}_{\mathbb{B}}-\aleph_{1.5}-$ c.c., and every poset with the $\operatorname{Add}_{\mathbb{B}}-\aleph_{1.5}-$ c.c. is proper and has the $\aleph_{2}-$ c.c.

It is easy to see that the proof of Lemma 3.7 can be adapted to show the following.

Proposition 3.10 For every set $X$ of ordinals, $\operatorname{Add}_{\mathbb{B}}(X)$ has the $\operatorname{Add}_{\mathbb{B}}-\aleph_{1.5}-$ c.c.

The proof of the following theorem is essentially contained in [3].

\footnotetext{
${ }^{2}$ For the purpose of this note, only.
} 
Theorem 3.11 (essentially [3]) (CH) Let $\kappa \geq \omega_{2}$ be a regular cardinal such that $\mu^{\aleph_{0}}<\kappa$ for all $\mu<\kappa$ and $\diamond\left(\left\{\alpha<\kappa \mid \operatorname{cf}(\alpha) \geq \omega_{1}\right\}\right)$ holds. Then there is a proper forcing notion $\mathcal{P}$ of size $\kappa$ with the $\aleph_{2}-c . c$. such that the following statements hold in the generic extension by $\mathcal{P}$.

(1) $2^{\aleph_{0}}=\kappa$

(2) $\operatorname{FA}\left(\left\{\mathcal{P} \mid \mathcal{P}\right.\right.$ has the $\operatorname{Add}_{\mathbb{B}}-\aleph_{1.5}-$ c.c. $\left.\}\right) \lambda$ for every $\lambda<\kappa$.

\subsection{A model of $\mathfrak{b}\left(\omega_{1}\right)=\aleph_{2}, \mathfrak{d}\left(\omega_{1}\right)$ large, and $\neg \mathrm{KA} \lambda$ for large $\lambda$}

The classical cardinal invariants for the continuum can be naturally extended to $\mathcal{P}(\kappa)$ or ${ }^{\kappa} \kappa$, for higher $\kappa$. For example, given a regular cardinal $\kappa$ one can define $\mathfrak{b}(\kappa)$ as the minimal size of a family $\mathcal{F}$ of functions $f: \kappa \longrightarrow \kappa$ such that no function $g: \kappa \longrightarrow \kappa$ dominates all $f \in \mathcal{F}$ modulo the ideal of bounded sets, which means that no $g$ as above is such that $\{v<\kappa \mid g(v) \leq f(v)\}$ is bounded for all $f \in \mathcal{F}$. With this definition, $\mathfrak{b}(\omega)$ is just the familiar bounding number $\mathfrak{b}$ on the Baire space. Similarly, we can define $\mathfrak{d}(\kappa)$ as the minimal size of a family $\mathcal{F}$ of functions $f: \kappa \longrightarrow \kappa$ such that for every $g: \kappa \longrightarrow \kappa$ there is some $f \in \mathcal{F}$ such that $\{v<\kappa \mid f(v) \leq g(v)\}$ is bounded in $\kappa \cdot \mathfrak{d}$ is then $\mathfrak{d}(\omega)$. Obviously, $\mathfrak{b}(\kappa) \leq \mathfrak{d}(\kappa)$ holds always for every $\kappa$.

Recall that, given an ideal $\mathcal{I}$ on a set $X, \operatorname{non}(\mathcal{I})$ is the least size of a subset of $X$ not in $\mathcal{I}$. An old observation of Rothberger ([12], see also [5], Theorem 2.8) is that $\mathfrak{b}=\operatorname{non}\left(\mathcal{K}_{\sigma}\right)$ and $\mathfrak{d}=\operatorname{cov}\left(\mathcal{K}_{\sigma}\right)$, where $\mathcal{K}_{\sigma}$ denotes the ideal on the Baire space $\sigma$-generated by the compact sets. Similar characterisations can be derived in general for $\mathfrak{b}(\kappa)$ and $\mathfrak{d}(\kappa)$ by the same proof:

Proposition 3.12 Given an infinite cardinal $\kappa, \mathfrak{b}=\operatorname{non}\left(\mathcal{K}_{\kappa, \kappa^{+}}\right)$and $\mathfrak{d}(\kappa)=$ $\operatorname{cov}\left(\mathcal{K}_{\kappa, \kappa^{+}}\right)$where, for all cardinals $\kappa, \lambda, \mathcal{K}_{\kappa, \lambda}$ denotes the ideal on ${ }^{\kappa} \kappa \lambda$-generated by the sets of the form $\left\{f \in{ }^{\kappa}{ } \mid f \leq g\right\}$ (for $g \in{ }^{\kappa}{ }$ ).

Given $f \in{ }^{\omega_{1}} \omega_{1}$, let $C_{f}$ be the club of $\omega_{1}$ whose enumerating function $\tilde{C}_{f}$ is such that $\tilde{C}_{f}(0)=f(0), \tilde{C}_{f}(n+1)=\tilde{C}_{f}(n)+f(n+1)$ for all $n<\omega$, and such that $\tilde{C}_{f}(v+1)=\tilde{C}_{f}(v)+f(v)$ if $v \geq \omega$. Clearly, the map $\Phi$ sending $f$ to $C_{f}$ is a bijection between ${ }^{\omega_{1}} \omega_{1}$ and $\mathcal{C}_{\omega_{1}}$ and $\left\{C_{f}: f \leq g\right\}$ is nowhere dense in $\tau_{\mathbb{B}}$ for all $g \in{ }^{\omega_{1}} \omega_{1}$. In fact, for every $p \in \mathbb{B}$ let $p^{\prime} \in \mathbb{B}$ extend $p$ and such that $\tilde{C}_{g}(\nu)=p^{\prime}(v)$ and $\tilde{C}_{g}(v+1)<p^{\prime}(v)$ for some $v$. Then no club of $\omega_{1}$ whose enumerating function extends $p^{\prime}$ belongs to $\left\{C_{f}: f \leq g\right\}$. But then, for every $\kappa, \Phi^{-1}(X) \notin \mathcal{K}_{\omega_{1}, \kappa}$ if $X \subseteq \mathcal{C}_{\omega_{1}}$ and $X \notin \mathcal{M}_{\mathcal{C}_{\omega_{1}}}^{\kappa}$. From this we immediately obtain the following inequalities (cf. for example [5], Proposition 5.5 for the corresponding $\mathfrak{b}=\operatorname{non}\left(\mathcal{K}_{\sigma}\right) \leq \operatorname{non}(\mathcal{M})$ and $\left.\operatorname{cov}(\mathcal{M}) \leq \operatorname{cov}\left(\mathcal{K}_{\sigma}\right)=\mathfrak{d}\right)$.

Proposition $3.13 \mathfrak{b}\left(\omega_{1}\right)=\operatorname{non}\left(\mathcal{K}_{\omega_{1}, \omega_{2}}\right) \leq \operatorname{non}\left(\mathcal{M}_{\mathcal{C}_{\omega_{1}}}^{\omega_{2}}\right)$ and $\mathfrak{d}=\operatorname{cov}\left(\mathcal{K}_{\omega_{1}, \omega_{2}}\right) \geq$ $\operatorname{cov}\left(\mathcal{M}_{\mathcal{C}_{\omega_{1}}}^{\omega_{2}}\right)$

A set $X$ of reals is called a Luzin set if it is uncountable and has countable intersection with all meagre sets of reals (see [9]). The same definition can be generalised to subsets of $\mathcal{C}_{\omega_{1}}$ : Let us say that $X \subseteq \mathcal{C}_{\omega_{1}}$ is Luzin if and only if it has cardinality at least $\aleph_{2}$ and has intersection of cardinality at most $\aleph_{1}$ with every $\omega_{2}$-meagre subset of $\mathcal{C}_{\omega_{1}}$. It is a 
well-known fact that if $G$ is $\operatorname{Add}\left(\omega_{1}\right)$-generic, then $\left\{r_{\alpha}^{G} \mid \alpha<\omega_{1}\right\}$ is a Luzin set in the extension (see for example [9]). This follows from the fact that $\operatorname{Add}\left(\omega_{1}\right)$ satisfies the relevant forms of clauses (2) and (4) from the introduction. By essentially the same argument we obtain the following.

Proposition 3.14 If $G$ is an $\operatorname{Add}_{\mathbb{B}}\left(\omega_{2}\right)$-generic, then $\left\{\cup \operatorname{range}\left(F_{\alpha}^{G}\right): \alpha<\omega_{2}\right\}$ is a Luzin set of clubs.

Proof Let $\left\{D_{\xi} \mid \xi<\omega_{1}\right\}$ be a collection of dense open subsets of $\mathcal{C}_{\omega_{1}}$. For every $\xi$ let $\left(p_{i}^{\xi}\right)_{i<\omega_{1}}$ be a sequence of conditions in $\mathbb{B}$ such that $D_{\xi}=\bigcup_{i<\omega_{1}}\left\{C \in \mathcal{C}_{\omega_{1}} \mid p_{i}^{\xi} \subseteq\right.$ $\tilde{C}$ \}. It suffices to show that there is some $\beta<\omega_{2}$ with the property that for $\alpha>\beta$ and all $\xi, p_{i}^{\xi} \subseteq F_{\alpha}^{G}$ for some $i$. But this is true since, by the $\aleph_{2}-$ c.c. of $\operatorname{Add}_{\mathbb{B}}\left(\omega_{2}\right)$, we may fix $\beta<\omega_{2}$ such that $\left(p_{i}^{\xi}\right)_{\xi, i<\omega_{1}} \in \mathbf{V}\left[G \cap \operatorname{Add}_{\mathbb{B}}(\beta)\right]$ and since each $F_{\alpha}^{G}$, for $\alpha>\beta$, is $\mathbb{B}$-generic over $\mathbf{V}\left[G \cap \operatorname{Add}_{\mathbb{B}}(\beta)\right]$ by Lemma 3.5 and Fact 3.4.

The main new consistency result in this note is the joint consistency of clauses (1)-(3) in the statement of Theorem 3.15.

Theorem 3.15 Let $\theta \geq \omega_{2}$ be a cardinal. Then the following statements hold after forcing with $\operatorname{Add}_{\mathbb{B}}(\theta)$.

(1) There is a Luzin subset of $\mathcal{C}_{\omega_{1}}$ of cardinality $\aleph_{2}$.

(2) $\mathfrak{m}(\mathbb{B}) \geq \theta$

(3) $\theta \leq 2^{\aleph_{0}} \leq 2^{\aleph_{1}} \leq \theta^{\aleph_{1}}$

Proof (1) follows immediately from Proposition 3.14 together with Lemma 3.5. (2) follows immediately from Lemma 3.6 together with Proposition 3.14, Lemma 3.5 and Fact 3.4: Given an $\operatorname{Add}_{B}(\theta)$-generic filter $G, \lambda<\theta$, and a collection $\mathcal{D}=\left\{D_{i} \mid i<\right.$ $\lambda\} \in \mathbf{V}[G]$ of dense subsets of $\mathbb{B}$ we may find $X \subseteq \theta,|X|=\max \left\{\lambda, \aleph_{1}\right\}$, such that $\mathcal{D} \in \mathbf{V}\left[G \cap \operatorname{Add}_{B}(X)\right]$. But then, if $\alpha \in \theta \backslash X, F_{G}^{\alpha}$ extends a condition in $D_{i}$ for every $i$.

$\operatorname{Add}_{\mathbb{B}}(\theta)$ adds at least $\theta$-many Cohen reals. In fact, let $\left(C_{\delta} \mid \delta \in \operatorname{Lim}\left(\omega_{1}\right)\right)$ be a ladder system and, for given $\operatorname{Add}_{\mathbb{B}}(\theta)$-generic filter $G$ and $\alpha<\theta$, let $f_{G}^{\alpha}: \omega \longrightarrow\{0,1\}$ be such that $f_{G}^{\alpha}(n)=0$ if and only if $\left|\left[F_{G}^{\alpha}(n), F_{G}^{\alpha}(n+1)\right) \cap C_{F_{G}^{\alpha}(\omega)}\right|$ is even. Then, a simple density argument shows that $f_{G}^{\alpha}$ is a Cohen real over $V\left[\left(f_{G}^{\alpha^{\prime}} \mid \alpha^{\prime}<\theta, \alpha^{\prime} \neq\right.\right.$ $\alpha)$ ]. That $2^{\aleph_{1}} \leq \theta^{\aleph_{1}}$ holds in the extension with $\operatorname{Add}_{\mathbb{B}}(\theta)$ follows easily from a simple counting argument of nice names for subsets of $\omega_{1}$ together with $\left|\operatorname{Add}_{\mathbb{B}}(\theta)\right|=\theta^{\aleph_{0}}$ and the $\aleph_{2}-$ c.c. of $\operatorname{Add}_{\mathbb{B}}(\theta)$.

Corollary 3.16 follows immediately from Theorem 3.15 together with the second part of Proposition 3.13 (for conclusion (1)), Proposition 2.6 and the first part of Proposition 3.13 (for conclusion (2)), and Proposition 2.2 (for conclusion (3)).

Corollary 3.16 Let $\theta \geq \omega_{2}$ be a cardinal. Then the following holds after forcing with $\operatorname{Add}_{\mathbb{B}}(\theta)$.

(1) $\mathfrak{d}\left(\omega_{1}\right) \geq \theta$

(2) $\operatorname{non}\left(\overline{\mathcal{M}}_{\mathcal{C}_{\omega_{1}}}^{\omega_{2}}\right)=\aleph_{2}$. In particular, $\mathfrak{b}\left(\omega_{1}\right)=\aleph_{2}$. 
(3) $\neg \mathrm{KA}_{\lambda}$ for every $\lambda<\theta$.

As we have seen in the above corollary, $\mathfrak{b}\left(\omega_{1}\right)=\aleph_{2}$ holds after forcing with $\operatorname{Add}_{\mathbb{B}}(X)$, for any set of ordinals $X$ of order type at least $\omega_{2}$. The equality $\mathfrak{d}\left(\omega_{1}\right)=\aleph_{2}-$ and therefore also $\mathfrak{b}\left(\omega_{1}\right)=\aleph_{2}$-follows of course from $2^{\aleph_{1}}=\aleph_{2}$ and can be easily forced in other ways too. For example it holds after forcing with any c.c.c. forcing over any model of $\mathfrak{d}\left(\omega_{1}\right)=\aleph_{2}$, simply because every function $f: \omega_{1} \longrightarrow \omega_{1}$ in the extension is dominated everywhere by a function $g: \omega_{1} \longrightarrow \omega_{1}$ in the ground model. Also, $\mathfrak{b}\left(\omega_{1}\right)=\aleph_{2}$ holds always after forcing with $\operatorname{Add}\left(\omega_{1}, \theta\right)$, for any $\theta \geq \omega_{2}$, where $\operatorname{Add}\left(\omega_{1}, \theta\right)$ is the forcing for adding $\theta$-many Cohen subsets of $\omega_{1}$ : We may assume $\mathbf{C H}$ in $\mathbf{V}$ since $\operatorname{Add}\left(\omega_{1}, \theta\right) \cong \operatorname{Add}\left(\omega_{1}, 1\right) \times \operatorname{Add}\left(\omega_{1}, \theta\right)$ and since $\operatorname{Add}\left(\omega_{1}, 1\right)$ forces $\mathrm{CH}$ and does not change $\operatorname{Add}\left(\omega_{1}, \theta\right)$. But then $\operatorname{Add}\left(\omega_{1}, \theta\right)$ has the $\aleph_{2}$-c.c., i.e., the relevant form of clause (2) from the introduction holds. This, together with the relevant form of clause (4) for $\operatorname{Add}\left(\omega_{1}, \theta\right)$, shows $\mathfrak{b}\left(\omega_{1}\right)=\aleph_{2}$ in the extension. Of course, $\diamond$ holds also in this extension. This, by Corollary 3.16, is in stark contrast to what holds after forcing with $\operatorname{Add}_{\mathbb{B}}(\theta)$ for $\theta \geq \omega_{2}$.

By essentially the same argument as in [13]—-showing that $\neg$ CG is preserved after adding Cohen reals—one can prove that $\operatorname{Add}(\omega, \theta)$ preserves $\neg \mathrm{CG}_{\lambda}$. Also, by refining the argument from [13], one can establish the following preservation result:

Lemma 3.17 For every cardinal $\theta, \operatorname{Add}(\omega, \theta)$ preserves $\neg \mathrm{KA}_{\lambda}$.

Proof Let $X$ be a set of ordinals and suppose that $\left\langle\dot{A}_{i} \mid i<\lambda\right\rangle$ is a sequence of $\operatorname{Add}(\omega, X)$-names for subsets of $\omega_{1}$ of order type $\omega$ such that some $p \in \operatorname{Add}(\omega, X)$ forces that $\left\{\dot{A}_{i} \mid i<\lambda\right\}$ witnesses $\mathrm{KA}_{\lambda}$. By homogeneity we may assume $p$ is the empty condition. The first observation is that for every $i$ there is a countable $Y_{i} \subseteq X$ such that $\dot{A}_{i}$ is in fact an $\operatorname{Add}\left(\omega, Y_{i}\right)$-name and such that for every $\alpha<\omega_{1}$ there is some $p \in \operatorname{Add}(\omega, X)$ such that $p \Vdash_{\operatorname{Add}(\omega, X)} \check{\alpha} \in \dot{A}_{i}$ if and only if there is some $p \in \operatorname{Add}\left(\omega, Y_{i}\right)$ such that $p \Vdash_{\operatorname{Add}\left(\omega, Y_{i}\right)} \check{\alpha} \in \dot{A}_{i}$.

For every $i$ let $\left(p_{n}^{i}\right)_{n<\omega}$ be an enumeration of $\operatorname{Add}\left(\omega, Y_{i}\right)$. Also, for every $n<\omega$, if there is some $\sigma \in \omega_{1}$ such that $p_{n}^{i} \Vdash_{\operatorname{Add}\left(\omega, Y_{i}\right)} \sup \left(\dot{A}_{i}\right)=\sigma$, then let $\mathcal{X}_{n}^{i}$ be a set of pairwise compatible $\operatorname{Add}\left(\omega, Y_{i}\right)$-conditions extending $p_{n}^{i}$ and such that $\{\xi<\sigma$ । $p \Vdash_{\operatorname{Add}\left(\omega, Y_{i}\right)} \xi \in \dot{A}_{i}$ for some $\left.p \in \mathcal{X}_{n}^{i}\right\}$ is cofinal in $\sigma$. The introduction of the $\mathcal{X}_{n}^{i}$, s is the new ingredient with respect to the proof in [13].

Given any club $C \subseteq \omega_{1}$ there are $i<\lambda, \sigma<\omega_{1}, \gamma<\sigma$ and $n^{C}<\omega$ such that $p_{n C}^{i}$ forces in $\operatorname{Add}\left(\omega, Y_{i}\right)$ that $\sup \left(\dot{A}_{i}\right)=\sigma$ and that $\left[\alpha, \alpha^{\prime}\right) \cap C \neq \emptyset$ for every two consecutive points $\alpha, \alpha^{\prime}$ of $\dot{A}_{i}$ above $\gamma$. Let $n^{*}<\omega$ be such that the set $\mathcal{C}$ of clubs $C$ such that $n^{C}=n^{*}$ is $\subseteq$-dense in the set of all clubs of $\omega_{1}$. For every $i<\lambda$, if there is a $\sigma$ such that $p_{n^{*}}^{i} \Vdash^{A} \operatorname{Add}\left(\omega, Y_{i}\right) \sup \left(\dot{A}_{i}\right)=\sigma$, then let $B_{i}=\left\{\xi<\sigma \mid p \Vdash^{\operatorname{Add}\left(\omega, Y_{i}\right)}\right.$ $\xi \in \dot{A}_{i}$ for some $\left.p \in \mathcal{X}_{n^{*}}^{i}\right\}$.

By $\subseteq$-density of $\mathcal{C}$ it suffices to show that if $C \in \mathcal{C}$, then there is some $i<\lambda$ such that $p_{n^{*}}^{i} \Vdash \sup \left(\dot{A}_{i}\right)=\sigma$ for some $\sigma$, and such that $\left[\beta, \beta^{\prime}\right) \cap C \neq \emptyset$ for every two consecutive points $\beta<\beta^{\prime}$ in $B_{i}$ above some $\gamma<\sigma$. But this is true by the previous paragraph and the definition of $B_{i}$ since all conditions in $\mathcal{X}_{n^{*}}^{i}$ extend $p_{n^{*}}^{i}$ and are pairwise compatible.

It follows from the above lemma that if we start with a model of $\neg \mathrm{KA}_{\aleph_{1}}+2^{\aleph_{1}}=$ $\aleph_{2}$ - which can be easily obtained by a countable support iteration of proper forcing 
or by [3] — and add any amount of Cohen reals to it, we will preserve both $\neg \mathrm{KA}_{\aleph_{1}}$ and $\mathfrak{d}\left(\omega_{1}\right)=\aleph_{2}$. In terms of obtaining $\mathfrak{b}\left(\omega_{1}\right)$ small together with strong failures of club guessing at $\omega_{1}$ (and together with $2^{\aleph_{1}}$ large), this is the best I can do without resorting to Theorem 3.15.

To finish this note, I will mention that I do not have at the moment any use for the homogeneity of $\operatorname{Add}_{\mathbb{B}}(X)$.

Open Access This article is distributed under the terms of the Creative Commons Attribution 4.0 International License (http://creativecommons.org/licenses/by/4.0/), which permits unrestricted use, distribution, and reproduction in any medium, provided you give appropriate credit to the original author(s) and the source, provide a link to the Creative Commons license, and indicate if changes were made.

\section{References}

1. Abraham, U., Shelah, S.: Forcing closed unbounded sets. J. Symb. Log. 48(3), 643-657 (1983)

2. Asperó, D., Mota, M.A.: Forcing consequences of PFA together with the continuum large. Trans. Am. Math. Soc. 367, 6103-6129 (2015)

3. Asperó, D., Mota, M.A.: A generalization of Martin's Axiom. Isr. J. Math. 210, 193-231 (2015)

4. Baumgartner, J.E.: Applications of the proper forcing axiom. In: Kunen, K., Vaughan, J.E. (eds.) Handbook of Set-Theoretic Topology, pp. 913-959. North Holland Publ. Co., Amsterdam (1984)

5. Blass, A.: Combinatorial cardinal invariants. In: Foreman, M., Kanamori, A. (eds.) Handbook of Set Theory. Springer, Berlin (2010)

6. Hamkins, J.D.: http://mathoverflow.net/questions/114590/forcing-with-product-vs-box-product (2012)

7. Ishiu, T.: The comparison of various club guessing principles. Ann. Pure Appl. Logic 166(5), 583-600 (2015)

8. Ishiu, T., Larson, P.: Some results about (+) proved by iterated forcing. J. Symb. Log. 77(2), 515-531 (2012)

9. Miller, A.: Special subsets of the real line. In: Kunen, K., Vaughan, J.E. (eds.) Handbook of SetTheoretic Topology, pp. 201-233. North Holland Publ. Co., Amsterdam (1984)

10. Miller, A.: Descriptive set theory and forcing. Lecture Notes in Logic. Springer, Berlin (1995)

11. Nyikos, P.: Antidiamond and anti-PFA axioms and topological applications [incomplete preliminary version]. http://people.math.sc.edu/nyikos/A.pdf

12. Rothberger, F.: Sur un ensemble toujours de première catégorie que est dépourvu de la propriété $\lambda$. Fund. Math. 32, 294-300 (1939)

13. Sakai, H.: Preservation of $\neg \mathrm{CG}$ by finite support product of Cohen forcing. http://www2.kobe-u.ac. $\mathrm{jp} / \sim \mathrm{hsakai} /$ Research/works.html

14. Shelah, S.: Cardinal Arithmetic, Oxford Logic Guides, vol. 29. Oxford University Press, Oxford (1994)

15. Shelah, S.: Proper and Improper Forcing. Perspectives in Mathematical Logic, vol. 29. Springer, Berlin (1998)

16. Zapletal, J.: Characterization of the club forcing. Papers on general topology and applications (Gorham, ME, 1995). Ann. NY Acad. Sci., vol. 806, pp. 476-484 (1996)

17. Zapletal, J.: A classification of definable forcings on $\omega_{1}$. Fund. Math. 153, 141-144 (1997)

18. Zapletal, J.: A dichotomy for forcing notions. Math. Res. Lett. 5, 213-226 (1998) 